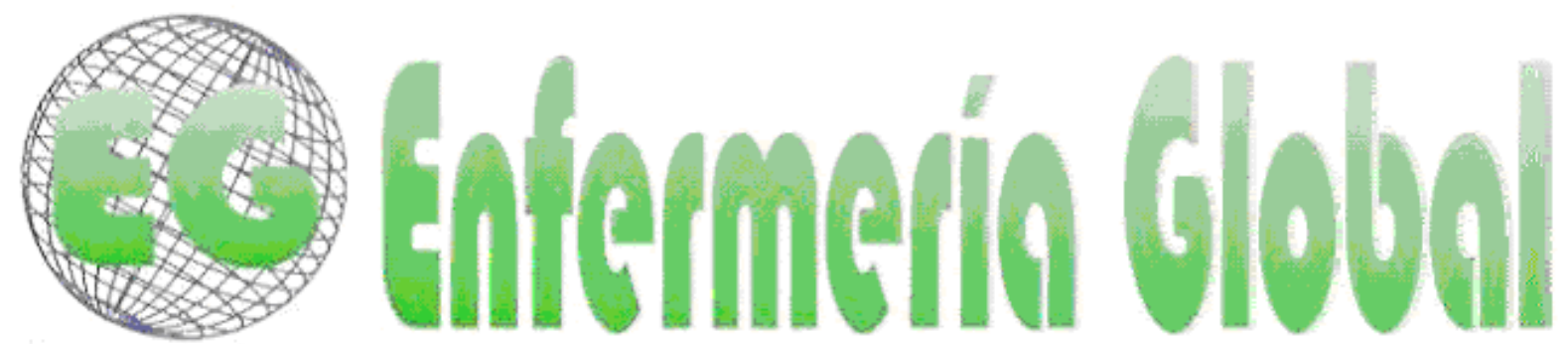

ISSN 1695-6141 Revista electrónica trimestral de Enfermería

N028

Octubre 2012

www.um.es/egloball

ENFERYERIA Y PERSPECTIVA DE GÉNERO

\title{
Evaluación de un programa de educación sexual sobre conocimientos y conductas sexuales en embarazadas
}

Evaluation of a sexual education program on knowledge and sexual behaviour in pregnant.

\begin{abstract}
*García González, S. **Duarte González, L. " ${ }^{* * *}$ Mejías Paneque, MC.
*Matrona. Hospital Juan Ramón Jiménez. Huelva. E-mail: zyrvita@hotmail.com **Matrona de Atención Primaria. Huelva. ${ }^{* * *}$ Matrona. Hospital Universitario Virgen Macarena. Sevilla.
\end{abstract}

Palabras clave: sexualidad; embarazo; programa; conocimientos; actitudes.

Keywords: sexuality; pregnancy; program; knowledge; attitudes.

\section{RESUMEN}

Objetivo: Evaluar el nivel de conocimientos de la sexualidad en la gestación previos y posteriores a un programa formativo implementado por un grupo de gestantes en el segundo y tercer trimestre.

Material y método: diseño cuasi-experimental pre-post test de un único grupo. Participaron 40 mujeres grávidas de bajo riesgo que se encontraban en su segundo y tercer trimestre de gestación. Todas eran de raza blanca. El $85 \%$ de las gestantes comprendían una edad entre 20 y 35 años, un $60 \%$ eran primigestas.

Resultados: al $60 \%$ de las mujeres del estudio les ha afectado el embarazo a su vida sexual. El trimestre que ellas creen que pueden disfrutar más del sexo, en un primer cuestionario el $62.5 \%$ contestaron que en el segundo, después de implantar el programa, el 95\% dieron esa respuesta. Entre las diferentes técnicas sexuales que existen, el $87.5 \%$ la conocían en un principio, después de realizar nuestro programa el porcentaje aumentó a un $97.4 \%$. En cuanto a los beneficios de los ejercicios de Kegel, en un primer cuestionario tan sólo el $25 \%$ sabían la respuesta correcta, mientras que más tarde aumentó a un $85 \%$ y su práctica era realizada un $12.5 \%$ antes de la intervención, y después un $40 \%$.

Discusión-Conclusión: después de aplicar nuestro programa específico se notaron algunas mejoras en el nivel de conocimientos y actitudes sexuales aunque debido probablemente a la pequeña muestra utilizada no se han encontrado resultados estadísticamente significativos salvo en una variable (práctica de Kegel).

\section{ABSTRACT}

Objective: Evaluate the level of knowledge of sexuality in pregnancy before and after a training program implemented by a group of pregnant women in the second and third trimesters.

Materials and methods: A quasi-experimental pre-post test single group. A total of 40 pregnant women at low risk who were in their second and third trimester were included. Regarding the ethnical group all 
women were white. The $85 \%$ of pregnant women included had an age between 20 and 35 years and for the $60 \%$ it was their first pregnancy.

Results: $60 \%$ of women have altered her sex life. The initially believed that the most suitable month to enjoy sex live more was on the second trimester, as shown on the first questionnaire $(62.5 \%)$. After implementing the program, these results increased up to the $95 \%$. Regarding the different sexual techniques, $87.5 \%$ knew them at first, after participating in our program this percentage increased to $97.4 \%$. In relation to the benefits of Kegel exercises, in the first questionnaire only $25 \%$ knew the correct answer, while after the program increased to $85 \%$. Its practice increased to $12.5 \%$ before the intervention, and then a $40 \%$ of pregnant women said they knew it and practiced it.

Discussion-Conclusion: after applying our specific program noted some improvements in the level of knowledge and sexual attitudes but probably due to the small sample used was not found statistically significant results except for one variable (practice Kegel).

\section{INTRODUCCIÓN}

Históricamente, la mujer embarazada en nuestra cultura se ha considerado un ser asexuado. La sexualidad ha sido frecuentemente ignorada en el diálogo entre la embarazada y su obstetra o se le han impuesto arbitrarios períodos de abstinencia. La confusión y la ansiedad que generan en la embarazada estas recomendaciones pueden llevar a limitar en forma extrema los contactos sexuales con su pareja o a no tenerlos y a generar agudos sentimientos de culpa. Ambas situaciones pueden alterar su relación de pareja durante el embarazo y durante un largo período posterior al mismo.

Se puede definir la sexualidad humana como las diferentes formas en que experimentamos y nos expresamos como seres sexuales. El conocimiento de nosotros mismos como mujeres $u$ hombres es parte de nuestra sexualidad, como es la capacidad que tenemos para las experiencias y respuestas eróticas. Nuestra sexualidad es una parte esencial de nosotros mismos, nos comprometamos o no en una relación sexual o en una fantasía sexual, o incluso si perdemos la sensibilidad de nuestros órganos genitales debido a una lesión ${ }^{(1)}$.

El estudio de la sexualidad humana es un objetivo de antropólogos, biólogos, investigadores médicos, sociólogos y psicólogos. Todas estas disciplinas hacen su contribución, porque la sexualidad humana refleja las capacidades biológicas, las características psicológicas y las influencias sociales y culturales. Los biólogos nos informan acerca de los mecanismos fisiológicos de la excitación y la respuesta sexual. La ciencia médica nos informa sobre las ETS y las bases biológicas de las disfunciones sexuales. Los psicólogos examinan cómo nuestra conducta sexual y nuestras actitudes son moldeadas por la percepción, el aprendizaje, el pensamiento, la motivación y la emoción, y la personalidad. Los sociólogos examinan las relaciones entre la conducta sexual y la religión, la raza y la clase social. Los antropólogos se centran en las similitudes y diferencias interculturales en la conducta sexual. La ciencia nos proporciona información, pero no puede tomar las decisiones sobre la conducta sexual por nosotros. Para tomar decisiones en lo sexual, debemos tener en cuenta nuestros valores ${ }^{(1)}$.

La condición sexuada del hombre es un fenómeno de extraordinaria amplitud, que caracteriza de un modo peculiar todos los estratos y componentes de la compleja unidad que constituye el hombre. La sexualidad no es sólo un fenómeno amplio en la constitución de lo personal, es un fenómeno extraordinariamente profundo, en cuanto 
que afecta al modo de ser de la persona, que aparece en todas las formas culturales, como un aspecto decisivo del modo de entenderse el hombre a sí mismo ${ }^{(2)}$.

La sexualidad comienza desde la propia gestación y termina con la muerte del individuo; impregna todos y cada uno de los actos de la vida cotidiana. Crea reglas de comportamiento, evoluciona con la historia y es una continua fuente de placer 0 frustración, desde la adolescencia hasta la vejez ${ }^{(2)}$.

Los tabúes, los prejuicios, la desinformación y muchas veces el desconocimiento alrededor de este tema que tanta atención precisa, han llevado a que se le investigue poco o se trate de forma equivocada ${ }^{(3)}$.

La mujer en la actualidad, mantiene una vida sexual activa, siempre que no existan tabúes sociales que la repriman o razones de salud que lo desaconsejen; aunque estudios realizados confirman que aproximadamente el $40 \%$ de las mujeres embarazadas manifiestan una disminución del deseo sexual, debido al temor infundado de causar daño al feto o al aborto espontáneo ${ }^{(2)}{ }^{(4)}$. En el segundo trimestre del embarazo se recupera el deseo sexual, asociándose en muchos casos a un mayor bienestar físico a la vez que decrecen los temores en relación a la pérdida del feto, siendo en este período cuando la mujer tiene una mayor lubricación vaginal, permitiendo más fácilmente el coito ${ }^{(6)}$.

Desde el punto de vista fisiológico y anatómico, se ha demostrado que el embarazo provoca un aumento de la irrigación vascular, un aumento de la sangre en los órganos pélvicos, debido a ello, a partir del segundo trimestre se reduce ostensiblemente el orificio vaginal, esta reducción del espacio anatómico de la vagina implica una mejor estimulación local y en consecuencia mejores posibilidades de satisfacción sexual ${ }^{(5)}$.

El embarazo y el parto son los principales factores de riesgo para desarrollar incontinencia urinaria (IU) en la mujer. La prevalencia de la IU tras el parto varía entre el 6 y el 43\%, según las investigaciones. Los ejercicios musculares del suelo pélvico, también llamados ejercicios de Kegel, sirven para reforzar la musculatura perivaginal y perianal, con el objeto de aumentar el control de las pérdidas de orina en la mujer ${ }^{(6)}$.

Considerando que existen modificaciones fisiológicas y psicológicas durante la gestación ${ }^{(7)}$, que afectan al comportamiento sexual de la pareja, los profesionales de la salud que trabajan en contacto directo con este grupo de población, que son la embarazada y su pareja, deben proporcionarle la información básica sobre la vida sexual durante y después de la gestación.

Teniendo en cuenta la necesidad de recibir información por parte de la embarazada $^{(8)(9)}$, la finalidad de este trabajo de investigación es evaluar un programa de educación sexual sobre los conocimientos y conductas sexuales en mujeres gestantes, esto incluye el comprender y reconocer por parte de ésta los cambios en la sexualidad que ocurren durante la gestación, asesorar sobre posibles riesgos de la actividad sexual, ofrecer la oportunidad a las parejas de expresar sus dudas y temores, proveer información acerca de la función sexual y ayudar con las disfunciones sexuales, entre otros.

Abordaremos este tema desde los centros de salud, en las clases de educación maternal. De esa forma captaremos el mayor número de embarazadas posibles para la implantación del programa de intervención con un seguimiento adecuado. 


\section{OBJETIVOS}

\section{General}

Evaluar el nivel de los conocimientos de la sexualidad en la gestación previos y posteriores a un programa formativo implementado por un grupo de gestantes en el segundo y tercer trimestre.

\section{Específicos}

- Identificar los conocimientos sobre sexualidad y embarazo en un grupo de gestantes durante el segundo y tercer trimestre.

- Comparar el nivel de conocimientos sobre la sexualidad durante la gestación antes y después de recibir un programa formativo específico.

\section{MATERIAL Y MÉTODO}

\section{Diseño y muestra}

Se trabajó con un diseño cuasi-experimental pre-post test de un único grupo. Se llevaron a cabo dos mediciones, una antes de implantar el programa y otra después de ésta. En el estudio participaron 40 mujeres grávidas de bajo riesgo que se encontraban en su segundo y tercer trimestre de gestación y que acudieron a clases de educación maternal en sus respectivos centros de salud, durante los meses de mayo y junio del 2010. Como otros criterios de inclusión se consideraron la voluntad de participar en el estudio, pertenecer al grupo de bajo riesgo obstétrico y saber leer y escribir en castellano,

La implantación del programa se realizó en tres centros de salud de la población de Huelva: Centro de Salud Molino de la Vega, C.S. Adoratrices y C.S. Torrejón.

El programa constaba de varias sesiones, cada una de ellas con una duración de dos a tres horas. Las clases se impartieron en aulas bastante amplias, que constaban de más de treinta sillas, una pizarra, un cañón y un ventilador (muy importante por el calor que padecen las embarazadas). Las mujeres disponían del material necesario para tomar anotaciones y rellenar los cuestionarios, y en determinados momentos de las clases se pasaron, a modo de ejemplo, objetos que se usan para el fortalecimiento del suelo pélvico como son los conos vaginales o las bolas chinas.

Entre los contenidos del programa caben destacar: la sexualidad humana, embarazo y sexualidad, adaptaciones biopsicológicas en el embarazo, respuesta sexual humana, cambios psicológicos durante la gestación, fortalecimiento del suelo pélvico, anatomía masculina y femenina, repercusión de la actividad sexual durante el embarazo y hemodinámica materna.

\section{Instrumento}

Un cuestionario estructurado con 15 preguntas cerradas de varias opciones y autocumplimentado, elaborado específicamente según el objetivo del estudio. El cuestionario fue sometido a una prueba de jueces en la que se incluyeron 
profesionales expertos en el tema (matronas y psicólogos), para garantizar la adecuación de los contenidos.

\section{Variables del estudio}

- Variables sociodemográficas: edad, primigestas, embarazo deseado.

- Variables obstétricas-ginecológicas: alto o bajo riesgo durante la gestación.

- Variables sobre hábitos sexuales: afectación del embarazo a la vida sexual, cambios del deseo sexual en el embarazo, otras técnicas sexuales, mayor apetencia sexual según trimestres de gestación.

- Variables sobre conocimientos sexuales y gestación: daño al bebé durante el coito, localización del tapón mucoso, si puede adelantar el parto el mantener relaciones sexuales, postura menos adecuada para mantener relaciones en el embarazo, beneficios de los ejercicios de Kegel y su práctica.

Este cuestionario se repartió al inicio de la sesión, después de la presentación por parejas, para obtener el grado de conocimientos acerca de la sexualidad durante el embarazo y sus actitudes sexuales antes de nuestra intervención. Cuando se concluyó la última sesión se pasó el mismo cuestionario para evaluar la eficacia de nuestro programa en la adquisición de conocimientos y conductas.

\section{Consideraciones éticas}

En todo momento se respetó la confidencialidad de los datos y las gestantes manifestaron su deseo de participación en el estudio pudiendo en cualquier momento abandonar el programa o no contestar a las preguntas del cuestionario, el cual era de carácter voluntario y anónimo.

\section{Análisis estadístico}

Para analizar los datos se utilizaron los procesos estadísticos del programa informático SPSS (versión 15.0). Se realizó una estadística descriptiva de la muestra del estudio. Para describir las distribuciones de datos se recurrió a calcular las proporciones y porcentajes relativos en cada una de las variables categóricas. Para relacionar dos variables dicotómicas en los diseños de antes y después, y de esa forma medir el cambio, utilizaremos el test de McNemar ${ }^{(10)}$.

\section{RESULTADOS}

Todas las participantes fueron de raza blanca. El 85\% de las gestantes comprendían una edad entre 20 y 35 años, un $60 \%$ eran primigestas, y de ellas, un $85 \%$ había sido un embarazo deseado.

En primer lugar, antes de comparar los datos de antes y después de la intervención, las gestantes reconocieron en un $60 \%$ que pensaban que el embarazo le había afectado a su vida sexual.

Se les cuestionó si pensaban que las relaciones coitales podían dañar a sus bebés. Antes de nuestra intervención el $90 \%$ respondieron que no, después de ésta, el porcentaje se incrementó a un 95\%. 
La siguiente pregunta hacía referencia a la localización del tapón mucoso. Al principio un $80 \%$ sabían la repuesta correcta, después de implantar nuestro programa, un $97.5 \%$.

En cuanto a los cambios en el deseo sexual de la gestante durante el embarazo hay que destacar que antes de nuestra implantación del programa, un $77.5 \%$ de las gestantes que formaron parte de nuestro cuestionario reconocían haber tenido cambios en el deseo, mientras que después de nuestra intervención fue un $90 \%$.

Seguidamente, a la pregunta de que si pensaban que tener relaciones sexuales podía adelantar el parto, un $87.5 \%$ respondieron que no en el primer cuestionario, mientras que la segunda vez que se realizó, después de nuestra intervención, el porcentaje aumentó a un $97.5 \%$.

La postura menos recomendada a partir de la semana 24-26 de embarazo es la de boca arriba. Cuando se les preguntó por ello antes de intervenir, un $65 \%$ supieron la respuesta correcta. Después de ésta, un $95 \%$ de las gestantes contestaron que no debían adoptar esa postura.

A la cuestión de si pensaban que siempre se puede mantener relaciones coitales durante el embarazo, en principio un $42.5 \%$ pensaban que no, frente a un $40 \%$ en el segundo cuestionario, con lo que no se apreció cambios importantes.

En lo referente al trimestre que ellas creen que pueden disfrutar más del sexo, en un primer momento el $62.5 \%$ de todas las mujeres que formaron parte del estudio respondieron que en el segundo trimestre (gráfico 1).

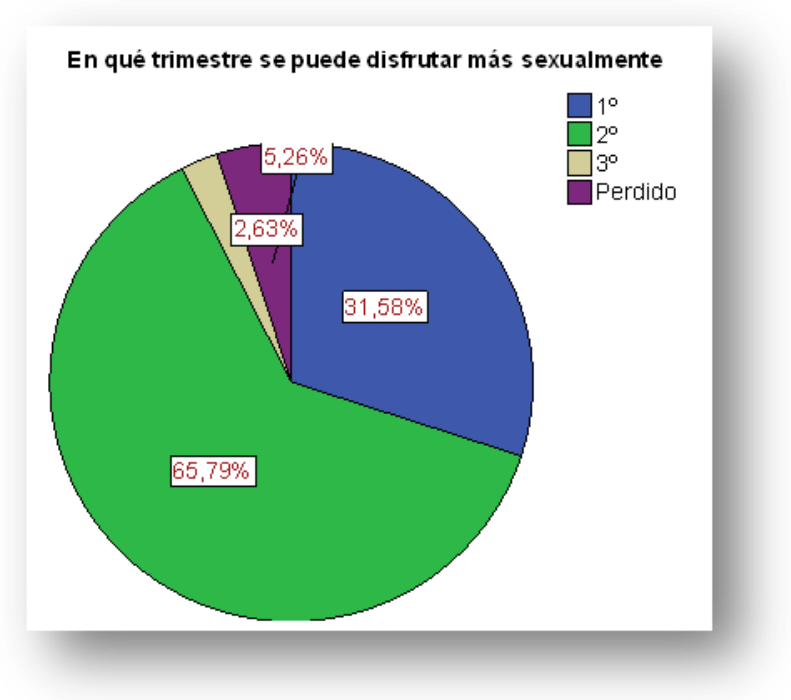

Después de implantar el programa, el 95\% dieron esa respuesta (gráfico 2). En los gráficos se muestran todos los porcentajes específicamente, incluyendo los perdidos.

Cuando se les preguntó sobre otras técnicas sexuales que pueden disfrutar, el $87.5 \%$ pensaron en la respuesta acertada. Después de realizar nuestro programa el porcentaje aumentó a un $\mathbf{9 7 . 4 \%}$. 


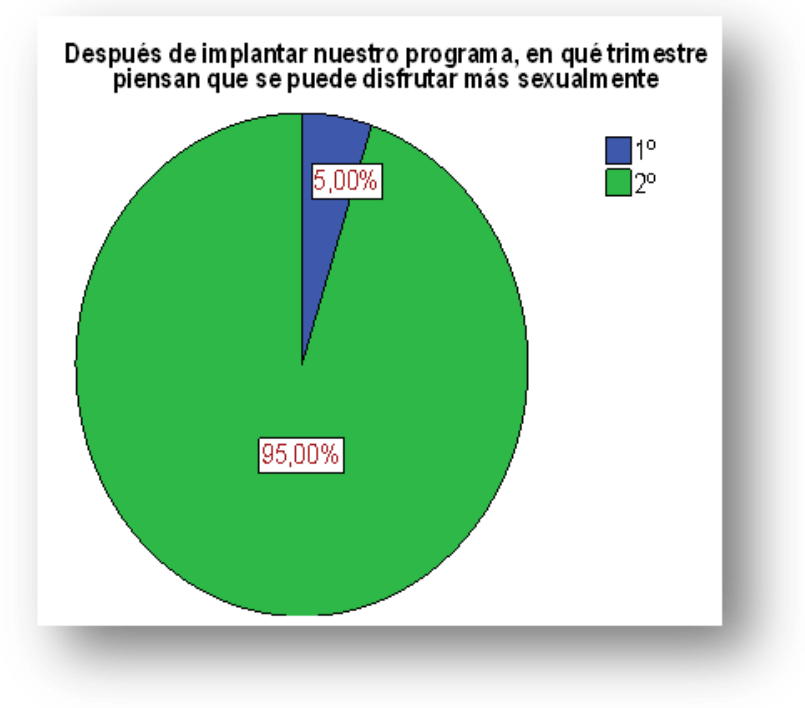

Los beneficios que se obtienen con los ejercicios de Kegel, en un primer cuestionario tan sólo el $25 \%$ sabían la respuesta correcta (gráfico 3), mientras que más tarde aumentó a un $85 \%$ (gráfico 4). Su práctica era realizada un $12.5 \%$ antes de la intervención (gráfico 5), y después un $40 \%$ de las gestantes afirmaban que sabían hacerlo y lo practicaban (gráfico 6).

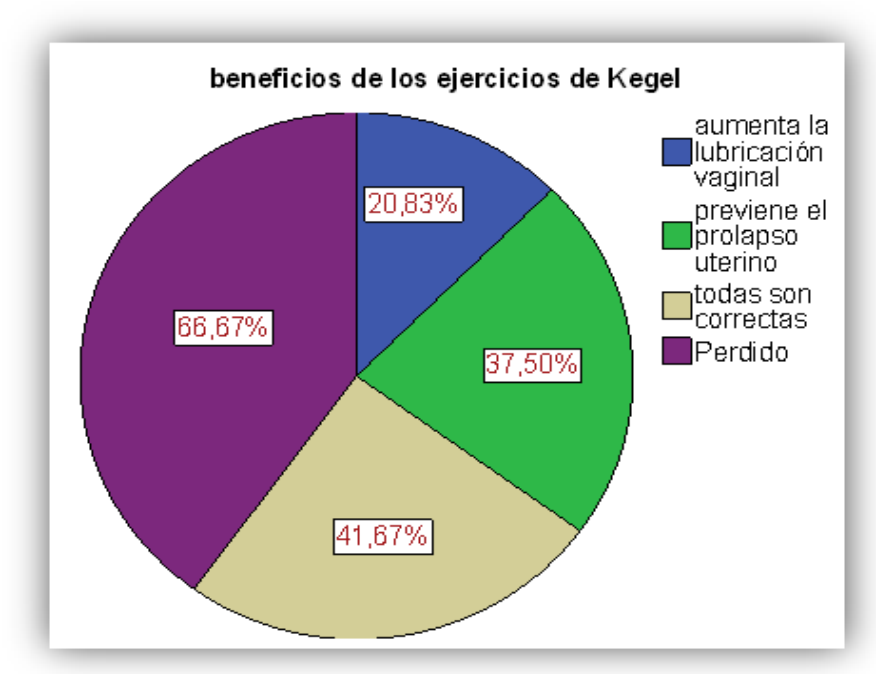


despúes de implantar nuestro programa, las mujeres que sabían cuáles eran los beneficios de los ejercicios de Kegel (la res puesta: todas son correctas)

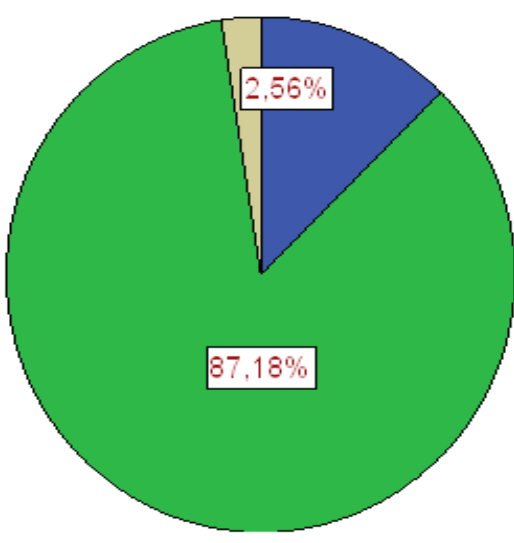

previene el

prolapso

uterino

$\square^{\text {todas son }}$

correctas

$\square$ perdido

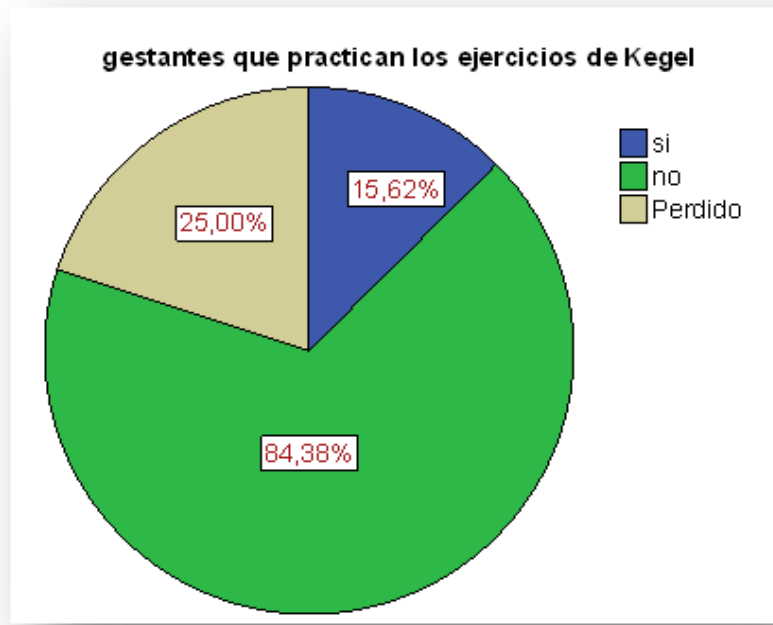

después de implantar nuestro programa, las gestantes que practican los ejercicios de Kegel

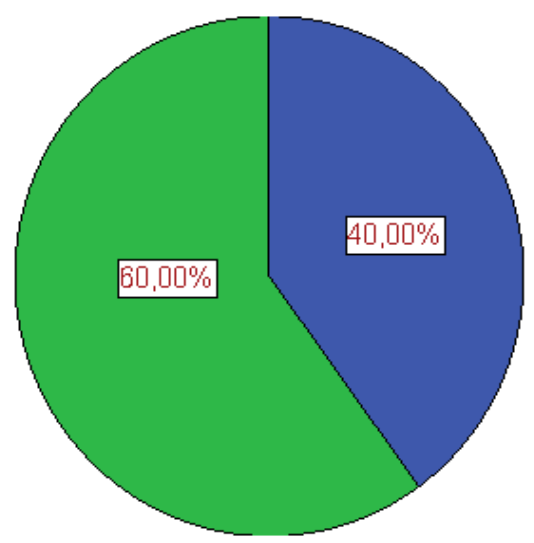

$\square$ si 
En nuestro estudio, se utilizó el test de McNemar ${ }^{(10)}$ para calcular el nivel de significación de las variables dicotómicas. Esta prueba se utiliza para analizar la relación entre dos variables dicotómicas relacionadas ya que contrasta los cambios de las respuestas utilizando la distribución de chi-cuadrado. Es útil para detectar cambios en las respuestas debido a una intervención, en este caso sería la implantación de nuestro programa.

Los resultados obtenidos con la aplicación del Test de McNemar, con un nivel de significación de $p=0,05$ han sido los siguientes:

- Para la variable de "práctica de los ejercicios de Kegel" $p=0,035$, con lo cual las mujeres que practicaban este tipo de actividades han cambiado significativamente en la segundo cuestionario.

- Para las demás variables no se han encontrado resultados estadísticamente significativos.

\section{DISCUSIÓN}

Durante el embarazo, y a medida que éste avanza, las funciones sexuales se reducen $^{(11)(12)}$ y la respuesta sexual se ve afectada como nos indican los cambios en los porcentajes, así como lo afirmaban algunas investigaciones comentadas al principio. La información proporcionada por profesionales parece insuficiente ${ }^{(13)}$ y después de aplicar nuestro programa específico se notaron algunas diferencias en los conocimientos y actitudes sexuales.

Llama la atención cómo en un primer cuestionario muchas de las preguntas no se contestaron (67 respuestas no se contestaron), quizás por timidez, ignorancia..., sin embargo, una vez que implantamos nuestro programa, las cuestiones que fueron dejadas en blanco disminuyeron notablemente, tan sólo 7 respuestas, lo cual demuestra, al menos, un gran cambio de actitud respecto a este tema, que hasta hace muy poco se consideraba como algo prohibido.

Se pudo observar que los conocimientos pre y post intervención se modificaron en gran medida si observamos los porcentajes. Una de las preguntas más notables sería la relacionada con los ejercicios de Kegel. Pese a la importancia que tiene el conocimiento de este tipo de ejercicios para el fortalecimiento de la musculatura pélvica en la embarazada, como así lo afirma un estudio sobre la efectividad de un programa de ejercicios musculares del suelo pélvico en la incontinencia urinaria después del parto ${ }^{(6)}$, muy pocas gestantes lo conocían cuando se les pasó el primer cuestionario y luego se incrementó notablemente su conocimiento y puesta en práctica llegando a ser estadísticamente significativo.

En cuanto a los conocimientos sobre otras técnicas sexuales, el trimestre donde se puede disfrutar más del sexo, la postura menos recomendada durante la gestación, la localización del tapón mucoso, si se le puede dañar al bebé, si siempre se pueden mantener relaciones sexuales o si éstas pueden adelantar el parto,... en todas ellas, la existencia de diferencias en los porcentajes de las respuestas entre el cuestionario pre y post intervención nos hace constar que nuestro programa ha sido relativamente efectivo.

En este estudio podemos encontrar varias limitaciones, como son: el bajo número de encuestadas, lo que supone poca representatividad; tres variables no dicotómicas lo que nos imposibilita utilizar el test de McNemar, con lo que se comparan con los 
porcentajes; entre las posibles opciones del cuestionario no se encuentra el ítem "no sabe, no contesta" lo que nos limita el tipo de conocimiento que pueden tener; y se requieren otras evaluaciones, con otros grupos, para poder generalizar el impacto de dicho programa.

Existen ciertos desconocimientos sobre las adaptaciones biopsicológicas durante el embarazo relacionadas con la sexualidad por lo que se debería priorizar el dar la información necesaria relacionada con ésta ${ }^{(14)}$. Los programas formativos específicos son un ejemplo de ello, y como hemos analizado en éste, pueden incrementar de una forma considerable los conocimientos y actitudes sexuales de la gestante.

Es necesaria una atención profesional orientada a aconsejar a las parejas acerca de los cambios psicosexuales y ayudarles a entender las fluctuaciones normales en el interés sexual durante el embarazo ${ }^{(15)}{ }^{(16)}$, todo ello con el objetivo de una mejor vida sexual, calidad y salud sexual de la pareja.

\section{CONCLUSIÓN}

Podemos afirmar que después de aplicar nuestro programa específico se notaron algunas mejoras en el nivel de conocimientos y actitudes sexuales aunque debido probablemente a la pequeña muestra utilizada no se han encontrado resultados estadísticamente significativos salvo en una variable (práctica de Kegel). Inicialmente existió un déficit de conocimientos importante y después se produjo un impacto positivo en éstos. Sería muy recomendable la implantación de programas formativos específicos de educación sexual en gestantes.

\section{Agradecimientos}

Al personal de los centros de salud del Molino de Vega, Adoratrices y Torrejón por hacer posible la implantación de nuestro programa.

\section{REFERENCIAS BIBLIOGRÁFICAS}

1. Spencer A, Nevid S, Fichner L, López F. Sexualidad humana. Pearson. 2005.

2. Hernández C. Educación en Sexología y sexualidad humana. Asociación Alcalá. 2005.

3. Trutnovsky G, Haas J, Lang U, Petru E. Women's perception of sexuality during pregnancy and after birth. Australian \& New Zealand Journal of Obstetrics \& Gynaecology. 2006; 46(4):282-7.

4. Bartellas E, Daley M, Bennett K. Sexuality and sexual activity in pregnancy. Department of obstetrics and Gynecology, Memorial University of Newfoundland, St. John's, Canada. 2000.

5. González I. Ginecología y sexualidad. Revista Cubana de Medicina General Integral. 2002; 18 (5).

6. Alonso L. Efectividad de un programa de ejercicios musculares del suelo pélvico en la incontinencia urinaria después del parto. Enfermería Clínica. 2007; 17 (5): 281-2.

7. Pauleta JR, Pereira NM, Graca LM. Sexualidad durante el embarazo. Journal of Sexual Medicine. 2010; (1): 136-142.

8. Heydari M, Asiabar A, Zade S. Couple's knowledge and attitude about sexuality in pregnancy. Tehran University Medical Journal. 2006; 64 (9): 83-89. 
9. Bratis L. La sexualidad humana durante el embarazo y el puerperio. Instituto de Sexología, Facultad de Medicina de la Universidad Charles. Praga, República Checa. 2009; 110 (7):427-31.

10. Martínez M.A. Test de McNemar para datos emparejados. Bioestadística amigable. 2006; 264-270.

11. Aslan G, Aslan D, Kizilyar A, Ispahi C, Esen A. A prospective analysis of sexual functions during pregnancy. International Journal of Impotence Research. 2005; 17(2):154-7.

12. Váldez E. Conceptos de sexualidad en perinatología. Revista chilena obstetricia Ginecología. 2003; 68 (4).

13. Savall A, Mendes A, Cardoso F. Pregnancy sexual behavior profile. Fisioterapia em Movimento, 2008 Apr-Jun; 21 (2): 61-70.

14. Wing Yee Fok, Louis YI-Si Chan, Pong Mo Yuen. Sexual behavior and activity in Chinese pregnant women. Acta Obstetricia \& Gynecologica Scandinavica. 2005; 84: 934-938.

15. Byrd J, Hyde J, Delamater J, Plant E. Sexuality during pregnancy and the year postpartum. Journal of Family Practice. 1998; 47.

16. Piñero $S$, Alarcos $G$, Arenas MT, Jiménez V, Ortega EM, Ramiro MJ. Características y modificaciones de la sexualidad durante el embarazo. NURE Inv. 2011 Ene-Feb; 8(50). 


\section{ANEXO 1. CUESTIONARIO}

\begin{tabular}{|c|c|c|c|c|}
\hline 1. ¿Cuántos años tienes? & a. $<20$ años & b. de 20 a 35 años & c. $>35$ años & \\
\hline ¿Es tu primer hijo? & a. sí & b. no & & \\
\hline 3. ¿Es un embarazo deseado? & a. sí & b. no & & \\
\hline $\begin{array}{l}\text { 4. ¿Tienes un embarazo de alto } \\
\text { riesgo? }\end{array}$ & a. sí & b. no & & \\
\hline $\begin{array}{l}\text { 5. ¿Crees que ha afectado el } \\
\text { embarazo a tu vida sexual? }\end{array}$ & a. sí & b. no & & \\
\hline $\begin{array}{l}\text { 6. ¿Piensas que las relaciones } \\
\text { coitales pueden dañar a tu } \\
\text { bebé? }\end{array}$ & a. sí & b. no & & \\
\hline $\begin{array}{l}\text { 7. ¿Dónde crees que está } \\
\text { situado el tapón mucoso? }\end{array}$ & a. en la vagina & $\begin{array}{l}\text { b. en el cuello } \\
\text { uterino }\end{array}$ & & \\
\hline $\begin{array}{l}\text { 8. ¿Has tenido cambios en el } \\
\text { deseo durante el embarazo? }\end{array}$ & a. sí & b. no & & \\
\hline $\begin{array}{l}\text { 9. ¿Piensas que tener } \\
\text { relaciones sexuales puede } \\
\text { adelantar el parto? }\end{array}$ & a. sí & b. no & & \\
\hline $\begin{array}{l}\text { 10. ¿Qué postura es la menos } \\
\text { recomendada a partir de la } \\
\text { semana } 24-26 \text { ? }\end{array}$ & a. de lado & b. boca arriba & & \\
\hline $\begin{array}{l}\text { 11. ¿Crees que siempre se } \\
\text { pueden mantener relaciones } \\
\text { coitales durante el embarazo? }\end{array}$ & a. sí & b. no & & \\
\hline $\begin{array}{l}\text { 12. ¿En qué trimestre crees que } \\
\text { se puede disfrutar más del } \\
\text { sexo? }\end{array}$ & a. en el primero & $\begin{array}{ll}\text { b. } & \text { en el } \\
& \text { segundo }\end{array}$ & $\begin{array}{l}\text { c. en el } \\
\text { tercero }\end{array}$ & \\
\hline $\begin{array}{l}\text { 13. Si el médico contraindica el } \\
\text { coito, ¿qué otras técnicas } \\
\text { sexuales puedes disfrutar? }\end{array}$ & a. masturbación & b. masaje erótico & c. sexo oral & $\begin{array}{l}\text { d. todas son } \\
\text { correctas }\end{array}$ \\
\hline $\begin{array}{l}\text { 14. ¿Qué beneficios se obtienen } \\
\text { con los ejercicios de Kegel? }\end{array}$ & $\begin{array}{l}\text { a. alivia el } \\
\text { estreñimiento }\end{array}$ & $\begin{array}{l}\text { b. previene el } \\
\text { prolapso uterino }\end{array}$ & $\begin{array}{l}\text { c. aumenta la } \\
\text { lubricación } \\
\text { vaginal }\end{array}$ & $\begin{array}{l}\text { d. todas son } \\
\text { correctas }\end{array}$ \\
\hline $\begin{array}{l}\text { 15. ¿Realizas los ejercicios de } \\
\text { Kegel? }\end{array}$ & a. sí & b. no & & \\
\hline
\end{tabular}

ISSN 1695-6141 